\title{
Redução da inflamabilidade da madeira de Pinus elliottii modificada com partículas de $\mathrm{TiO}_{2}$
}

\section{Reduction of flammability of Pinus elliottii wood modified with $\mathrm{TiO}_{2}$ particles}

Paula Zanatta ${ }^{1}$, Matheus Lemos Peres ${ }^{1}$, Ezequiel Gallio ${ }^{1}$, Debora Duarte Ribes ${ }^{1}$, Marília Lazarotto ${ }^{2}$, Darci Alberto Gatto ${ }^{3}$, Mário Lúcio Moreira ${ }^{4}$

\author{
${ }^{1}$ Programa de Pós Graduação em Ciência e Engenharia de Materiais, Universidade Federal de Pelotas, Pelotas, Rio \\ Grande do Sul, RS, Brasil. \\ e-mail: paulazanatta236@gmail.com; matheusldeperes@gmail.com; egeng.florestal@gmail.com; dribes22@gmail.com. \\ ${ }^{2}$ Faculdade de Agronomia, Departamento de Horticultura e Silvicultura, Universidade Federal do Rio Grande do Sul, \\ Porto Alegre, RS, Brasil. \\ e-mail: marilia.lazarotto@ufrgs \\ ${ }^{3}$ Centro de Engenharias, Engenharia Industrial Madeireira, Universidade Federal de Pelotas, Pelotas, RS, Brasil. e-mail: \\ darcigatto@yahoo.com \\ ${ }^{4}$ Instituto de Física e Matemática, Departamento de Física, Universidade Federal de Pelotas, Pelotas, RS, Brasil. e-mail: \\ mlucio3001@gmail.com
}

\section{RESUMO}

A madeira é um material formado basicamente por polímeros, os quais são pouco resistentes a altas temperaturas e suscetíveis ao fogo. Em aplicações é desejável que esta madeira tenha um melhor desempenho quanto à ignição e perda de resistência mecânica devido à degradação térmica de seus componentes. $\mathrm{O}$ aumento de desempenho pode ser obtido pela modificação das propriedades térmicas madeira através de seu tratamento. Assim, utilizou-se o processo solvotermal assistido por micro-ondas para a modificação da superfície e do interior da madeira de Pinus elliotttii com $\mathrm{TiO}_{2}$ visando o aumento da resistência à inflamabilidade e à degradação térmica pelo retardamento da carbonização. Para isso, foi preparada uma solução precursora alcoólica com isopropóxido de titânio. Com temperatura de $140^{\circ} \mathrm{C}$, por 60 minutos, foram cristalizadas, na superfície interna e externa da madeira, nanopartículas de $\mathrm{TiO}_{2}$ na fase anatase. Utilizando a Microscopia Eletrônica de Varredura foi possível demostrar o recobrimento uniforme e homogêneo. Através do espectro no infravermelho por Transformada de Fourier verificaram-se indícios de ligação por ponte de hidrogênio entre os dois materiais. A formação de uma camada protetora com as partículas proporcionou maior estabilidade e resistência térmica devido ao caráter cerâmico das nanopartículas de $\mathrm{TiO}_{2}$. A partir das análises termogravimétricas foi determinada uma perda de massa de $70 \%$ aos $380^{\circ} \mathrm{C}$ e $11 \%$ de resíduos aos $700^{\circ} \mathrm{C}$, diferentemente da madeira modificada que nestas temperaturas atingiram percentagens de $45 \%$ e $46 \%$, respectivamente. Em adição, verificou-se pelos testes de chama, retardo em cerca de 7 vezes o tempo para a ignição da madeira modificada, quando comparada com o controle. Desse modo, pode-se afirmar que a modificação da madeira pelo processo solvotermal assistido por micro-ondas foi efetiva na redução de sua inflamabilidade bem como de sua degradação com o aumento da temperatura. Cita-se ainda, que o método se apresenta com grande potencial para o aumento da qualidade da madeira e consequentemente de suas aplicações.

Palavras-chave: teste de chamas; degradação térmica; sistema micro-ondas.

\begin{abstract}
Wood is composed by polymeric materials, with fire susceptibility. For applications, wood with better ignition resistance and lower fire stenght reduction is desirable. Enhancing of wood thermal properties can be achieved by means of right modification of wood by treatment. Thus, the microwave-assisted solvotermal process was implemented to modify the surface of the Pinus elliotttii wood with $\mathrm{TiO}_{2}$ in order to increase the resistance to flammability and thermal degradation through the retardation of carbonization. An alcoholic precursor solution with titanium isopropoxide was prepared and nanoparticles of $\mathrm{TiO}_{2}$ were crystallized to anatase on wood surface and interior at $140^{\circ} \mathrm{C}$, for 60 minutes. Using Scanning Electron Microscopy, it was possible to demonstrate the uniform and homogeneous coating, and by Fourier transform infrared
\end{abstract}


spectroscopy, it was possible to indicate presence of hydrogen bonds between the two materials. The formation of protective particle layer afforded greater stability and thermal resistance due to the ceramic character of $\mathrm{TiO}_{2}$. From the thermogravimetric analysis, $70 \%$ of weigh loss at $380^{\circ} \mathrm{C}$ and $11 \%$ of residual content at $700^{\circ} \mathrm{C}$ was verified, differently from the modified wood that at these temperatures reached $45 \%$ and $46 \%$, respectively. Corroborating with these results, it was evident from the flame tests that wood with the presence of particles delayed about 7 times the moment of ignition. Thus, the microwave-assisted solvotermal process has great potential to transform wood and other porous materials into a less flammable material, maintaining its entire structure for a longer time when exposed to adverse conditions

Keywords: flame test; termal degradation; microwave system.

\section{INTRODUÇÃO}

A madeira como um recurso renovável e abundante, apresenta propriedades diversificadas e boas condições de resistência mecânica associada à baixa ou alta massa específica. Além disso apresenta absorção acústica sendo um material de engenharia amplamente empregado na construção, decoração e mobiliário. Contudo, considerando uma aplicação sob temperaturas elevadas, por ser um material polimérico suscetível ao fogo, apresenta limitações no seu uso quando em contato com chama ou fonte de calor de alta intensidade [1,2].

Quando comparada a outro material, a madeira se destaca dentre outras características pela sua resistência ao calor. Porém, por se tratar de um combustível sólido, há possibilidade de queima através de reações pirolíticas, oxidativas e hidrolíticas [3]. Assim, os polímeros da parede celular da madeira (celulose, hemicelulose e lignina) sofrem modificações, originando uma mistura de vapor de água, compostos voláteis, alcatrões, gases, fumaça e carvão altamente reativo, em diferentes faixas de temperatura [4,5], causando impacto ao ambiente e alterando as propriedades originais do material.

Para controlar a vulnerabilidade à degradação térmica e a combustão, várias abordagens foram desenvolvidas e empregadas em aplicações práticas. Produtos ignífugos ou retardadores de chama revestidos superficialmente, incorporados ou impregnados na madeira, formam uma camada protetora que evita a propagação superficial da chama ao se fundir com a elevação da temperatura, retarda o tempo de ignição e reduz a taxa de liberação de calor [1-3,6,7]

Muitas pesquisas têm examinado a eficácia de retardadores de fogo, dentre eles: solução contendo uréia [8], boro [9], fósforo [10] e partículas inorgânicas [11], cada um com suas próprias vantagens e limitações em termos de eficácia, custos e impactos ambientais. Especialmente, a modificação com materiais inorgânicos em escala nanométrica é considerada promissora para aumentar a resistência da madeira ao fogo $[1,12]$. Dentre as estruturas nanométricas utilizadas para revestir a superfície da madeira, o $\mathrm{TiO}_{2}$ têm sido investigado, pois acredita-se que os efeitos sinérgicos resultantes das interações com a matriz madeira, são capazes de proporcionar propriedades térmicas mais estáveis [1,11]. Este é caracterizado por se apresentar em diferentes fases (anatase, rutilo e brookita), ser um óxido semicondutor com gap de 3,2 eV, estável e quimicamente inerte [13,14].

A aplicação de um processo termal assistido por micro-ondas para modificar a madeira de modo a revestir e incorporar materiais inorgânicos, como o $\mathrm{TiO}_{2}$, na superfície e no interior se torna interessante devido a metodologia direta. Esse, pouco relatado para esta finalidade, oferece vantagens em relação aos demais processos, pois pode diminuir custos e etapas envolvidas no processo. Além disso, o consumo de energia e o tempo para produção das partículas são reduzidos e há minimização na produção de resíduos [1519]. A ação das micro-ondas na estrutura da madeira, através do alinhamento das moléculas pelo campo elétrico, pode vir a facilitar a deposição uniforme e criar sítios para nucleação e crescimento do $\mathrm{TiO}_{2}$.

Assim, visando um método mais amigável ambientalmente e associado a um material inorgânico de baixa toxicidade, o objetivo deste trabalho foi verificar o efeito do retardamento da ignição e, consequentemente, da degradação térmica da madeira de Pinus elliottii modificada pela cristalização de partículas de $\mathrm{TiO}_{2}$ por meio do método solvotermal assistido por micro-ondas.

\section{MATERIAIS E MÉTODOS}

\subsection{Material}

A modificação da superfície foi implementada em amostras de Pinus elliottii altamente porosas, oriundas de um povoamento homogêneo da Região Sul do Rio Grande do Sul. As dimensões definidas para o processo foram de $20 \times 20 \times 20 \mathrm{~mm}$ em umidade de equilíbrio de 12\%. Para a produção das partículas inorgânicas de $\mathrm{TiO}_{2}$, utilizou-se isopropóxido de titânio $\left(\mathrm{Ti}\left[\mathrm{OCH}\left(\mathrm{CH}_{3}\right)_{2}\right]_{4}\right)$ e álcool etílico $\left(\mathrm{C}_{2} \mathrm{H}_{6} \mathrm{O}\right)$ de 97 e $95 \%$ de pureza, respectivamente. 


\subsection{Modificação da madeira}

O método solvotermal assistido por micro-ondas foi o utilizado para modificar a madeira por meio da cristalização de partículas de $\mathrm{TiO}_{2}$. Para isso, preparou-se uma solução alcoólica de $30 \mathrm{~mL}$ com $0.07 \mathrm{~mol}^{-1}$ isopropóxido de titânio, sob constante agitação e fluxo de nitrogênio, na qual estavam inseridas as amostras de madeira. Então, transferiu-se a solução precursora, contendo a amostra de Pinus elliottii, para uma célula reacional de teflon e acoplada em um sistema micro-ondas. A modificação da madeira ocorreu a $140^{\circ} \mathrm{C}$ por 60 minutos, utilizando uma taxa de aquecimento de $6^{\circ} \mathrm{C} /$ minuto. As amostras permaneceram em estufa a $100^{\circ} \mathrm{C}$ por 24 horas para secagem.

\subsection{Caracterização}

\subsubsection{Difração de Raios-X (DRX)}

Para verificar a estrutura cristalina formada na superfície da madeira devido a parte inorgânica após a síntese foi utilizado um difratômetro de anodo rotatório marca Bruker, modelo D8 Advance, radiação $\mathrm{Cu} \mathrm{K} \alpha(\lambda$ $=1,5406 \AA$ A), com tensão $40 \mathrm{kV}$ e $150 \mathrm{~mA}$ em um intervalo de 10 a $90^{\circ}$ no modo $2 \theta$ com abertura da fenda de divergência de $0,5^{\circ}$ e da fenda de recepção de $0,3^{\circ}$, usando passo de $0,02^{\circ}$ com acumulação de 1 s/ponto.

\subsubsection{Análise morfológica}

Para analisar a forma e a distribuição de tamanho das partículas de $\mathrm{TiO}_{2}$ na superfície e no interior da madeira, bem como o grau de homogeneidade do processo de modificação após as condições aplicadas no processo, em diferentes magnificações, foi utilizado um Microscópio Eletrônico de Varredura JEOL JSM 6610LV (MEV). Este equipamento é acoplado com Espectrômetro de Energia Dispersiva de Raios X (EDX), o qual permitiu realizar a detecção e mapeamento dos elementos químicos presentes na amostra.

\subsubsection{Espectroscopia no Infravermelho por Transformada de Fourier (FT-IR)}

As alterações químicas na superfície decorrentes das condições atribuídas no processo de modificação da madeira foram avaliadas por meio de um Espectrômetro de Infravermelho com Transformada de Fourier (RT FTIR, Shimadzu Prestige21 Spectrometer, Shimadzu, Japão). As análises foram configuradas para efetuar 32 varreduras de transmitância, resolução de $4 \mathrm{~cm}^{-1}$ e leituras entre 4000 e $600 \mathrm{~cm}^{-1}$.

\subsubsection{Análise térmica}

A verificação do comportamento da madeira modificada sob elevada temperatura foi realizada pela análise termogravimétrica (TGA) e as respectivas derivadas (DTG), através do equipamento TGA-60 da Shimadzu, a partir de $30^{\circ} \mathrm{C}$ até a temperatura máxima de $700^{\circ} \mathrm{C}$, com taxa de aquecimento de $10^{\circ} \mathrm{C} /$ minuto, sob atmosfera de gás nitrogênio.

\subsubsection{Teste de retardamento de chamas}

$\mathrm{O}$ retardamento de chamas foi avaliado qualitativamente e quantitativamente nas amostras de madeira expostas diretamente a uma chama aberta. Para esse teste, as amostras, em triplicata, foram fixadas a 10 centímetros de uma haste de aço inoxidável acoplada em uma balança de precisão, a qual continha um bico de Bunsen conectado com o gás combustível butano. Por meio da variação do peso verificou-se a massa residual no decorrer do tempo, até que as amostras atingissem $20 \%$ do seu peso inicial. Em um mesmo momento, foi conferida a facilidade de ignição das amostras e registrou-se, por meio de imagens, o processo de propagação da chama.

\section{RESULTADOS}

\subsection{Caracterização da madeira modificada com $\mathrm{TiO}_{2}$}

Na Figura 1 é possível visualizar os poros dispostos na seção transversal (a,b), longitudinal tangencial (c,d) e a superfície do substrato. Na amostra controle a parede celular e a superfície são livres de recobrimento inorgânico, ao passo que na superfície modificada com $\mathrm{TiO}_{2}$ por meio sistema solvotermal assistido por micro-ondas, há presença de estruturas inorgânicas depositadas na parede celular externa do substrato, formando uma camada de proteção. 

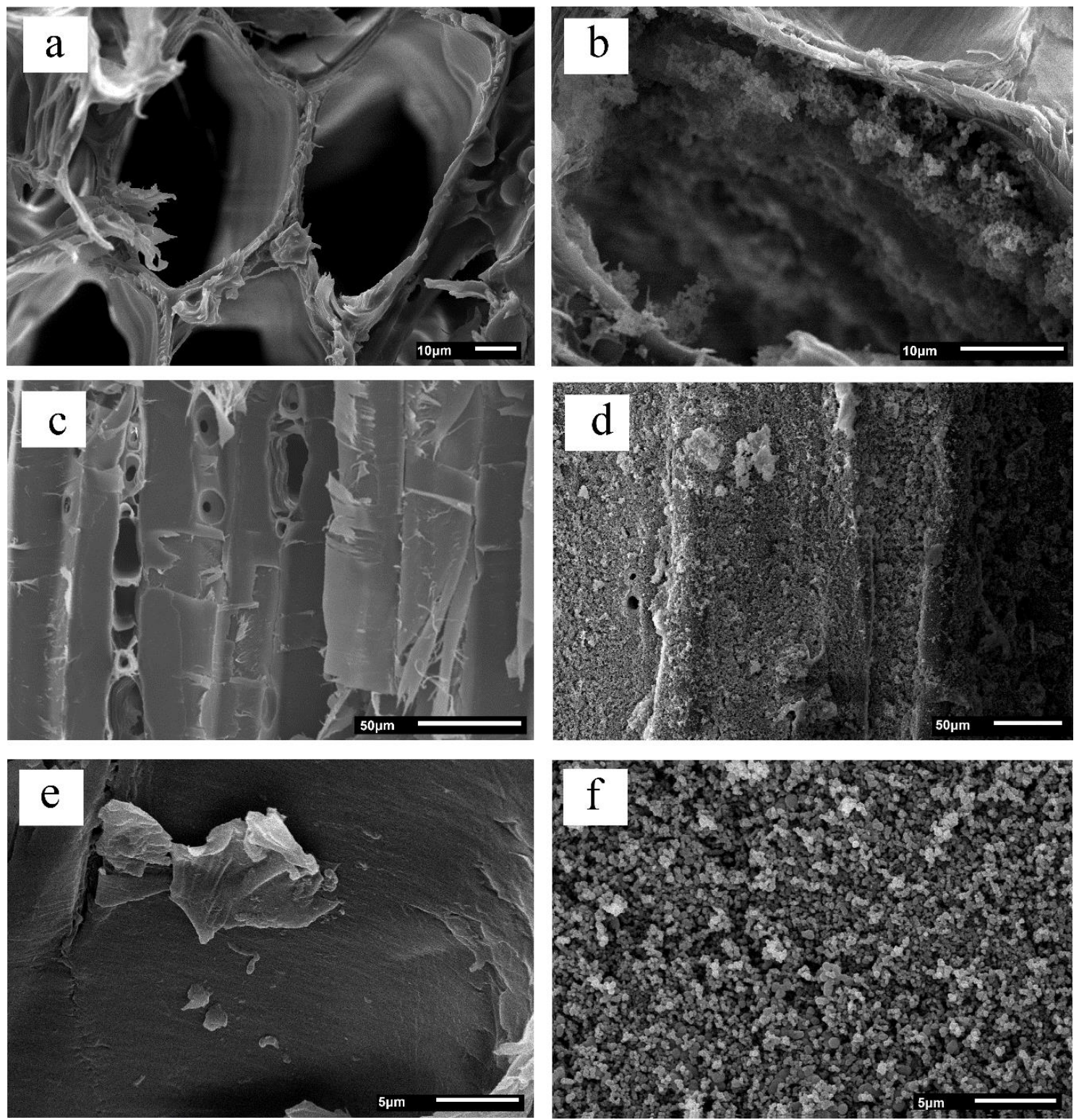

Figura 1: Microscopia Eletrônica de Varredura da madeira controle (a, c, e) e madeira modificada com $\mathrm{TiO}_{2}$ por meio do método solvotermal assistido por micro-ondas $(b, d, f)$.

A modificação por meio da cristalização de partículas de $\mathrm{TiO}_{2}$ é baseada na nucleação e crescimento destas na parede celular da madeira. Segundo KONG et al. [20] a superfície e o interior do lúmen servem como sítios de nucleação, onde sob condições hidrotérmicas permitem o posterior crescimento das partículas cerâmicas. Sabe-se que a estabilização de um núcleo, para posterior crescimento, passa por uma minimização de energia livre, a fim de atingir um raio crítico, a partir do qual o crescimento se dará possivelmente pelo processo de Ostwald Ripening. Assim, a ancoragem do titânio sobre a superfície ou sobre as paredes do traqueídeo facilita o processo, pois oferece um ponto de nucleação. Como as taxas de nucleação deste processo são altas, visto que a cristalização é rápida, a distribuição do tamanho das partículas tende a ser homogêneo na superfície da madeira. A Figura 1 demonstra que a solução precursora, tempo e temperatura oferecidos são ideais para um revestimento homogêneo e uniforme na matriz. RASSAM et al. [21] encontraram resultados muito semelhantes, contudo a solução precursora utilizada foi $\mathrm{TiCl}_{4}$ que exige expressivos cuidados na manipulação, o que torna o processo mais oneroso e perigoso. Já SUN et al. [22] utilizou um processo hidrotermal que necessitou de diferentes etapas para uma deposição homogênea e WANG et al. [23] levaram de 3-5 dias para finalizar o processo, tempo significativamente superior ao exposto neste estudo.

A madeira utilizada é porosa, composta de estruturas com diâmetro na ordem de micrômetros. As amostras modificadas não apresentaram seus lúmens totalmente preenchidos, contudo a parede celular 
interna destes e a superfície foi revestida com partículas aglomeradas semiesféricas na ordem de $200 \mathrm{~nm}$. Tal fenômeno dificultou ou praticamente impossibilitou a exposição direta da matriz ao ambiente externo. Em observância ao espectro EDX, juntamente com o mapeamento realizado, verificou-se que as partículas depositadas na superfície são formadas a partir do elemento Ti e que estes elementos também estão presentes nos interstícios da parede celular (Figura 2).
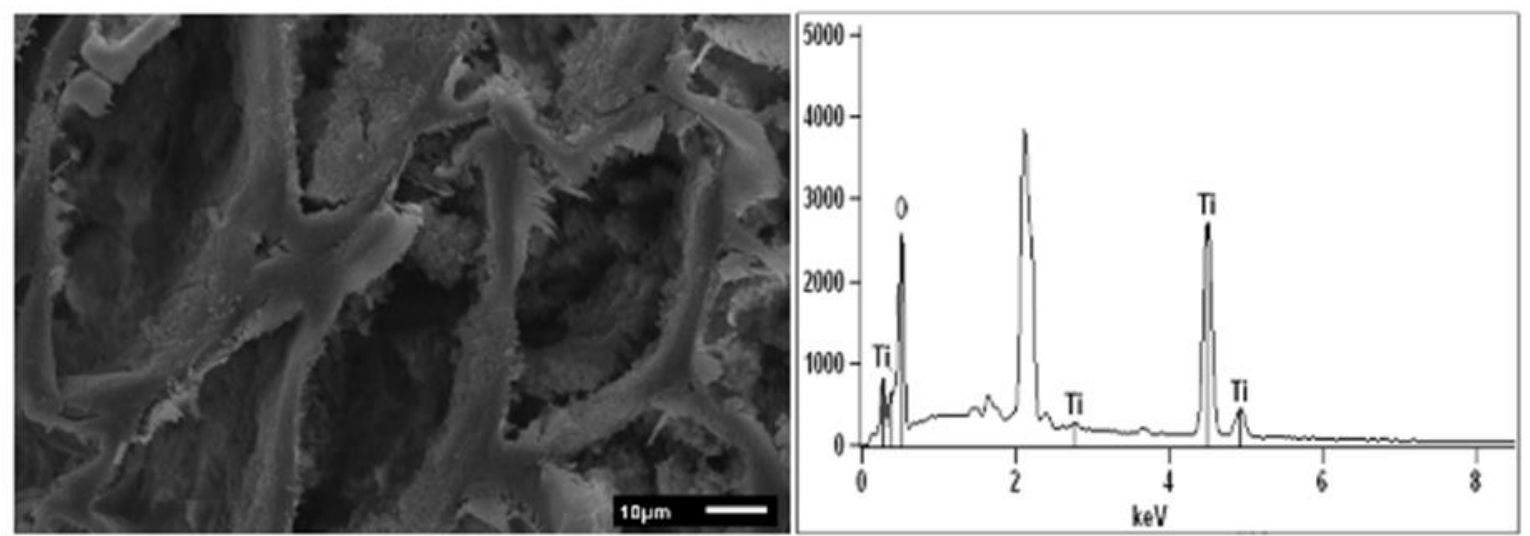

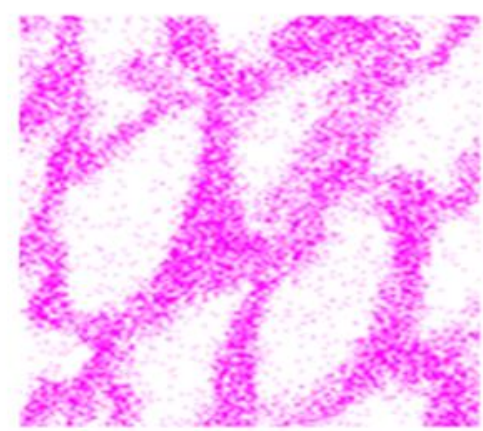

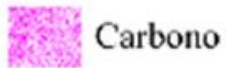

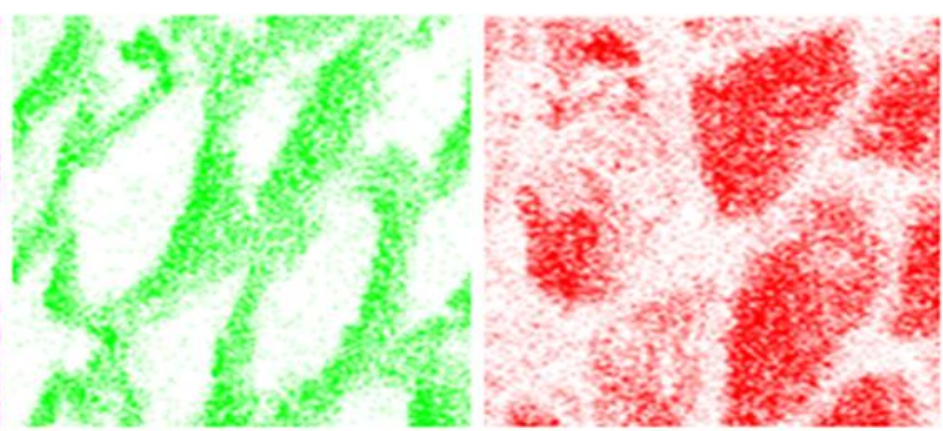

Oxigênio

\section{Titânio}

Figura 2: Espectro e mapeamento EDX na seção transversal da madeira modificada com $\mathrm{TiO}_{2}$ por meio do método solvotermal assistido por micro-ondas.

Uma vez confirmada a deposição de $\mathrm{TiO}_{2}$ na parede celular da madeira, a melhora nas suas propriedades fica condicionada a morfologia do material depositado. Cristalinidade e pureza, bem como a fase do material são de grande importância na melhora ou até produção de uma propriedade específica.

De modo a elucidar a influencia da morfologia, a análise dos espectros de difração mostra que a superfície da madeira está recoberta por partículas de $\mathrm{TiO}_{2}$ majoritariamente na fase anatase, com pequena fração de rutilo (Figura 3). Estas fases cristalinas são representadas por picos $25,3^{\circ}, 48,3^{\circ}, 53,9^{\circ}$ e $75,6^{\circ}$, e pelo pico $44,9^{\circ}$, verificados nos padrões das fichas cristalográficas indexadas JCPDS 21-1272 e JCPDS 211276 , respectivamente. Os picos de difração $15^{\circ}$ e $22,7^{\circ}$ são característicos da celulose cristalina presente na madeira, fortemente reduzidos para a madeira modificada. Por outro lado, o pico em $34,7^{\circ}$ ficou mais evidente, o que demonstra a eficácia da síntese solvotermal assistida por micro-ondas em alterar estrutura da madeira. Tal fato se dá em razão da propriedade característica marcante do método em recobrir totalmente a superfície dificultando assim a detecção da celulose do pico 22,7 . Além da qualidade da deposição e homogênea nucleação, o processo se dá em tempo reduzido quando comparado com a literatura, o que oferece grande vantagem tanto na pesquisa quanto em aplicações. 


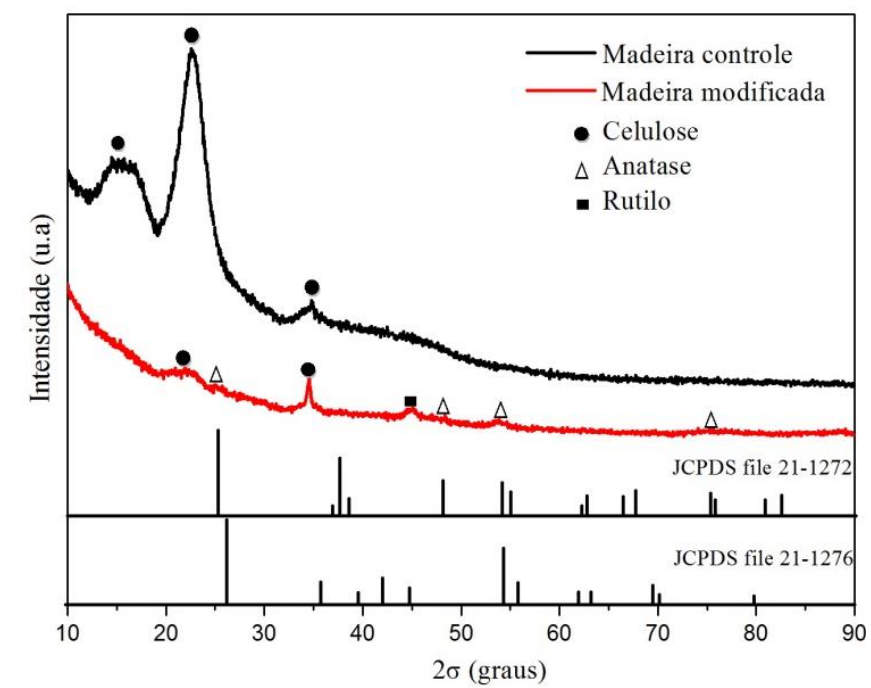

Figura 3: Difratograma de Raios- $\mathrm{X}$ da madeira controle e da madeira modificada com $\mathrm{TiO}_{2}$ por meio do método solvotermal assistido por micro-ondas.

A espectroscopia no infravermelho permitiu verificar as alterações dos grupos funcionais da madeira após o processo de modificação (Figura 4). A semelhança visual detectada nos espectros se dá pela análise de duas amostras de base madeira. Entretanto verifica-se fenômenos de redução, aparecimento e deslocamentos de modos vibracionais evidentemente em decorrência da deposição pelo método utilizado. Picos reduzidos ou não detectados na madeira modificada sugerem que as condições oferecidas pela síntese das partículas podem modificar a superfície da madeira, alterando ou formando alguns compostos.

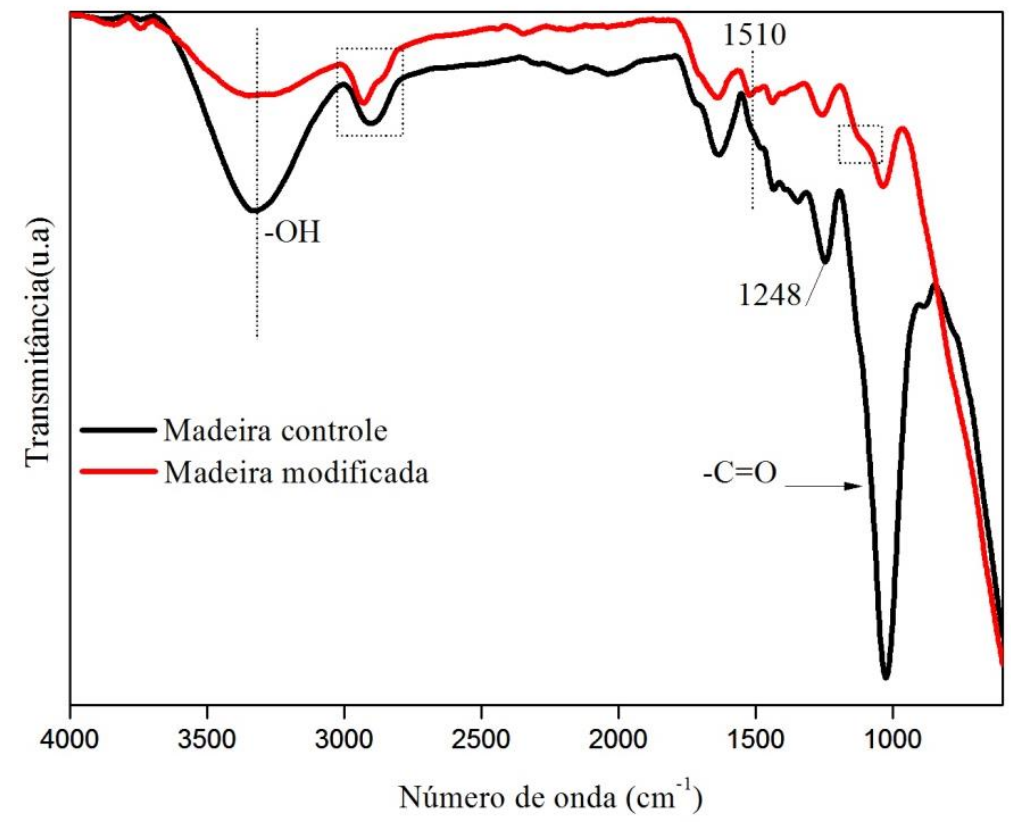

Figura 4: Espectro Infravermelho (FT-IR) da madeira controle e madeira modificada com $\mathrm{TiO}_{2}$ por meio do método solvotermal assistido por micro-ondas.

É expressiva a redução dos picos em $1026 \mathrm{~cm}^{-1}$ e $1248 \mathrm{~cm}^{-1}$ na madeira modificada, os quais representam o estiramento simétrico $\mathrm{C}=\mathrm{O}$ nas xilanas (carboidrato encontrado na hemicelulose) e associação $\mathrm{OH}$ na celulose e hemiceluloses, respectivamente [24,25]. Quando a madeira foi submetida ao tratamento solvotermal assistido por micro-ondas, iniciaram-se processos simultâneos de modificação da madeira, enfraquecimento de algumas ligações presentes nos polímeros, bem como favorecimento de outras, o que se mostrou evidente em comparação com as características originais do material.

A vibração observada na região entre $3060 \mathrm{~cm}^{-1}$ a $3520 \mathrm{~cm}^{-1}$ é referente as vibrações dos grupos hidroxila $\left(\mathrm{OH}^{-}\right)$presentes na parede celular da madeira [26]. A redução nesta faixa de transmitância demonstra redução da quantidade de tais grupos interativos, indicando possíveis criações de sítios favoráveis 
para a nucleação de $\mathrm{TiO}_{2}$. Houve alteração nas bandas situadas em $2910 \mathrm{~cm}^{-1}$ e $2865 \mathrm{~cm}^{-1}$ referentes as vibrações C-H. A alteração espectral na região de $1120 \mathrm{~cm}^{-1}$ indica que, além do recobrimento com as partículas e ligações do tipo ponte de hidrogênio, houve reações entre o precursor e a madeira com ligações Ti-O-C, igualmente ao encontrado por WANG et al. [23], que afirmam que isto ocorre pela reação Ti-OH e madeira-OH.

\subsection{Análise Térmica}

Análises térmicas baseadas em gravimetria informam o comportamento quantitativo da degradação e produção de carvão de um dado material com a mudança de temperatura. Através destas, pode se ter conhecimento do potencial de um produto retardador de chamas, o qual é avaliado por meio da secagem e formação de gases voláteis e resíduos durante e após a medição [5,7].

As curvas TG e DTG obtidas da madeira controle e modificada com $\mathrm{TiO}_{2}$ são mostradas na Figura 5. Conforme descrito na literatura, é comum este material apresentar três estágios de degradação térmica, começando pela: hemicelulose, celulose e lignina, sucessivamente, além da perda inicial de água presente na parede celular [27]. De maneira geral a madeira controle perdeu $8 \%$ de massa até atingir $230^{\circ} \mathrm{C}$, a qual foi atribuída a evaporação da água de impregnação e carbonização parcial da hemicelulose, já que esta começa a decompor a partir de $180^{\circ} \mathrm{C}$ e, neste momento, a pirólise é lenta e há formação de gases voláteis não inflamáveis. O segundo estágio ocorreu entre $230-380^{\circ} \mathrm{C}$ e foi marcado pela máxima pirólise em $362^{\circ} \mathrm{C}$ (curva DTG), onde a madeira sofreu uma degradação de $60 \%$ de sua estrutura física, advinda de reações que originam gases voláteis inflamáveis e degradam principalmente o polímero celulose. Acompanhando o processo, a lignina segue a degradar gradual e lentamente, devido a sua complexidade e reticulação, e então aos $700^{\circ} \mathrm{C}$ forma resíduos de carbono com massa de $11 \%$.
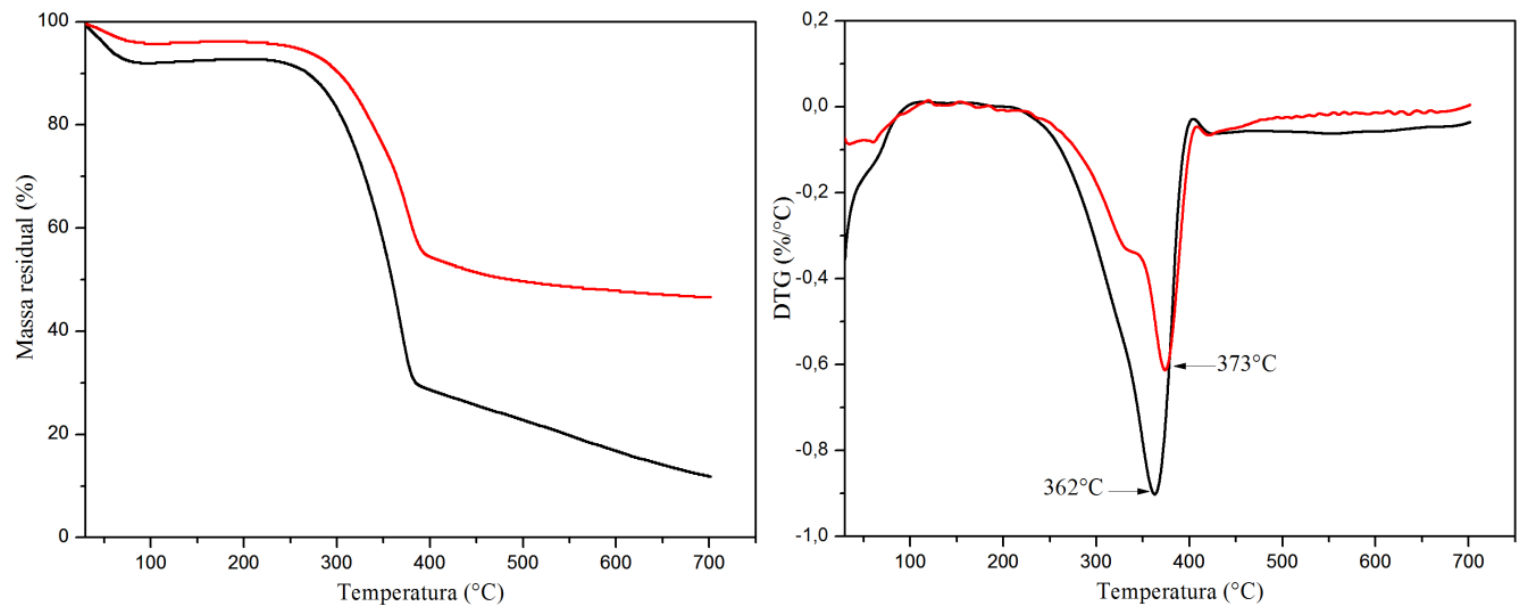

- Madeira controle

Madeira modificada

Figura 5: Análise térmica (TGA e DTG) da madeira controle e da madeira modificada com $\mathrm{TiO}_{2}$ por meio do método solvotermal assistido por micro-ondas.

O comportamento das amostras estudadas foi semelhante, contudo, a madeira modificada teve menor taxa de degradação e demonstrou ter o maior pico endotérmico deslocado para uma temperatura mais elevada, quando comparada à controle. $\mathrm{O}$ método proposto de deposição e incorporação de partículas tornou a madeira mais estável termicamente. Isso se deu pela interação entre o $\mathrm{TiO}_{2}$, depositado superficialmente em grande quantidade, e os radicais $\mathrm{OH}-$ dos polissacarídeos, o que dificultou o acesso do calor e do gás oxigênio aos constituintes químicos que compõem a madeira.

Além disso, foi verificado que é necessária maior energia para decompor a madeira modificada, visto que aos $700^{\circ} \mathrm{C}$ cerca de $46 \%$ de resíduo ainda estava presente. Isto indica que o processo executado atua como retardador e tem potencial para diversas aplicações. A necessidade de maior energia para degradação da madeira modificada tem sua razão na interação química entre a nanopartícula cerâmica depositada e a parede celular, proporcionada pelo método utilizado, a qual é vencida com muito mais dificuldade quando comaprada com a amostra controle. A madeira modificada por $\mathrm{TiO}_{2}$ pelos métodos de WANG et al. [23] e LU e HU [28], "sol-gel" e "camada a camada", respectivamente, ao atingir $700^{\circ} \mathrm{C}$ apresentavam cerca de 
$20 \%$ de resíduos, significativamente inferior ao visto na curva TG, o que comprova maior eficiência devido a interação mais estável entre os materiais pelo método proposto no presente trabalho.

Além disso, o processo de deposição do $\mathrm{TiO}_{2}$ pelo método utilizado pode ter favorecido a formação de char (carvão vegetal), que por apresentar ligações carbono-carbono de estrutura grafítica, são impossíveis de se degradar por pirólise, necessitando de temperaturas de até $3000^{\circ} \mathrm{C}$. Segundo LOWDEN e HULL [7], a formação de char tem efeito significante na degradação da madeira, retardando a mesma. O carvão vegetal formado não exerce função direta de resistência na madeira, mas funciona como uma barreira entre o interior composto por polímeros inalterados e a frente de pirólise, diminuindo a taxa de transmissão de calor. Desse modo, pode-se afirmar que tais mecanismos reduzem a taxa de degradação da madeira e aumentam a formação de carvão vegetal.

\subsection{Retardamento de chamas}

As amostras revestidas com $\mathrm{TiO}_{2}$ apresentaram um retardo na queima ao serem expostas à chama aberta de gás butano (Figura 6). Estas perderam $80 \%$ da massa inicial após 10 minutos em média, diferentemente da madeira controle que atingiu este valor na metade do tempo, em aproximadamente 5 minutos. Acredita-se que as partículas que revestem a superfície da madeira juntamente com uma possível formação de char atuam como obstáculos para a liberação de calor, e para o acesso do gás oxigênio. Assim, os gases voláteis formados com o aquecimento são liberados lentamente, explicando este retardamento e a perda menos abrupta da massa em relação ao tempo. Estes resultados se assemelham ao encontrado por SUN et al. [1], que ao recobrirem a madeira combinando $\mathrm{TiO}_{2}$ e $\mathrm{ZnO}$, verificaram tempo prolongado para a combustão e inibição da produção de fumaça, o que também se aplica ao presente estudo.

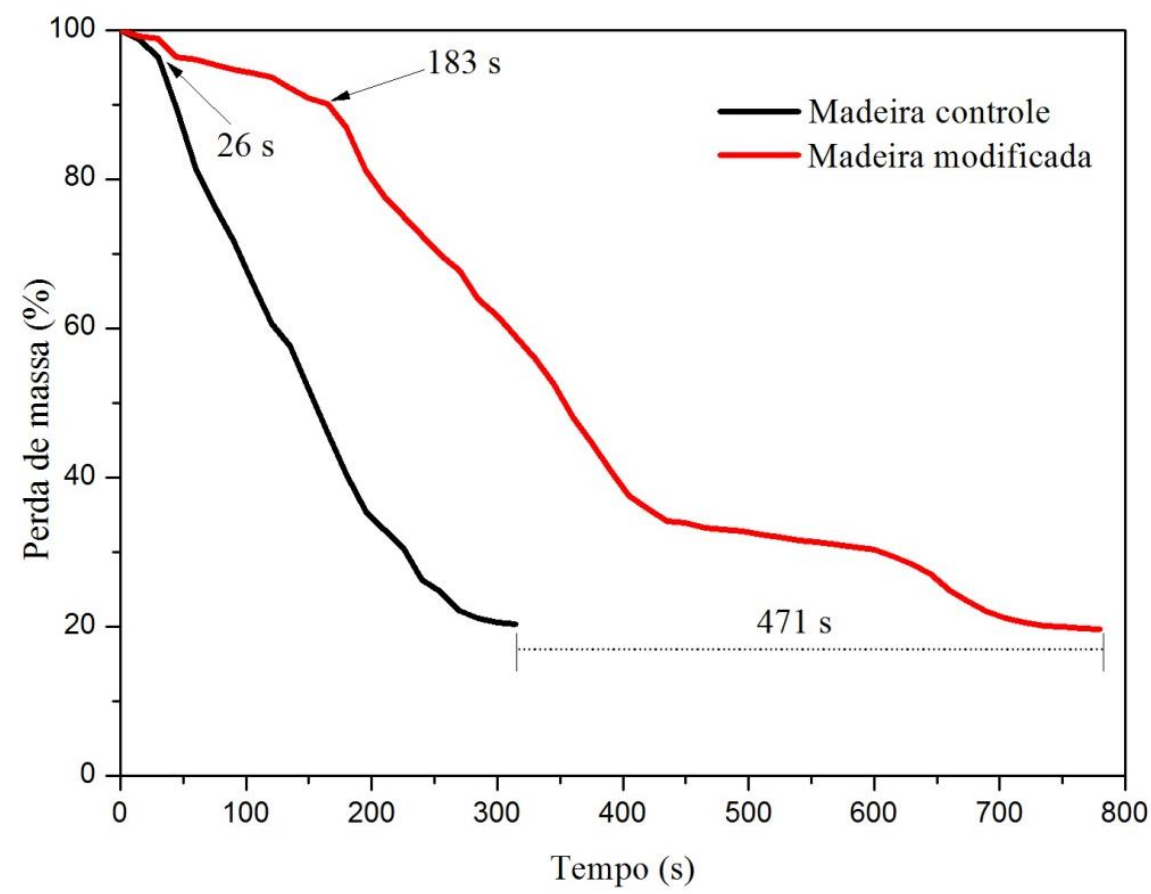

Figura 6: Comportamento das madeiras controle e modificada com $\mathrm{TiO}_{2}$ por meio do método solvotermal assistido por micro-ondas no ensaio teste de retardamento de fogo

A madeira controle demonstrou facilidade em acender uma chama piloto (média de 26 segundos), a qual deu início ao processo de queima e se propagou rapidamente (Figura 7a). Por outro lado, para a madeira modificada com $\mathrm{TiO}_{2}$, a ignição iniciou em aproximadamente 183 segundos, e teve a chama apagada no decorrer do tempo, o que contribuiu para transformá-la em um material menos inflamável. Esta afirmação é visível em 450 segundos de exposição ao fogo, em que houve uma alteração na curva representada pela linha vermelha da Figura 6. Quando o recobrimento deixou de criar uma barreira para a liberação dos gases inflamáveis decorrentes do aquecimento, o Ti presente nos microespaços da parede celular, foi fundamental para uma propagação do fogo em velocidade reduzida. 
(a)

(b)

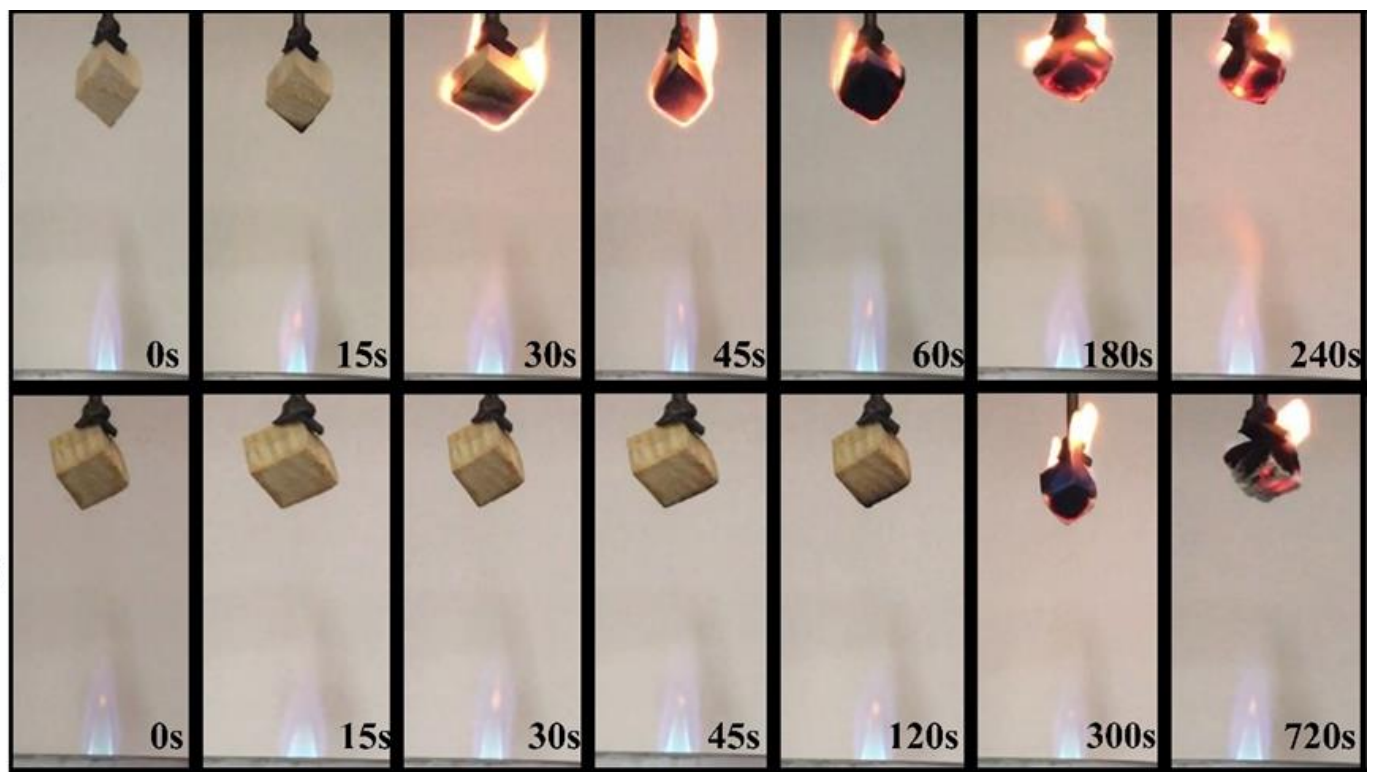

Figura 7: Propagação do fogo observada em diferentes tempos no teste de retardamento de chama da madeira controle (a) e madeira modificada com $\mathrm{TiO}_{2}$ por meio do método solvotermal assistido por micro-ondas (b).

Conforme visualizado, o revestimento homogêneo, a incorporação de partículas de $\mathrm{TiO}_{2}$ nas condições proporcionadas na síntese e uma possível formação de char dificultaram as reações químicas e as misturas inflamáveis que aceleram a combustão e a degradação da madeira. A união de um material de origem polimérica com uma cerâmica em escala nano/micrométrica torna-se potencial como retardador de fogo para madeira, principalmente quando o processo utilizado é rápido, simples e não faz uso de reagentes químicos tóxicos ao ambiente.

Em resposta aos perigos apresentados pela toxicidade, fumaça e corrosividade de compostos retardadores de fogo comerciais, como o boro e compostos halogenados [7,26], as partículas inorgânicas utilizadas como produto não geram gases tóxicos e ainda possibilitam preservar as características originais da madeira por um maior período de tempo quando exposta a condições de alta temperatura.

\section{CONCLUSÕES}

Foi possível revestir e incorporar de forma homogênea e uniforme partículas inorgânicas de $\mathrm{TiO}_{2}$ na estrutura física da madeira em etapas e tempo reduzidos. As condições oferecidas não afetaram a porosidade do material e proporcionaram interação entre a cerâmica e a matriz orgânica, de modo que as propriedades relacionadas a degradação térmica e combustão da madeira original fossem melhoradas.

Dessa forma conclui-se que a estabilidade sob condições de calor e a condição de menor inflamabilidade atribuída pela proteção do revestimento, indicam que a metodologia aplicada neste estudo é adequada para produção de madeira com comportamento controlável e projetado, que consequentemente, agrega valor e otimiza o seu emprego. Além disso o caráter não tóxico do dióxido de titânio agrega ainda mais valor ao efeito de retardamento no que se refere a propagação da chama.

Salienta-se, por fim, que os resultados obtidos podem ser sensivelmente diferentes para madeira de Pinus elliottii proveniente de diferentes regiões, visto que a genética, procedência e as condições oferecidas para o desenvolvimento da árvore são fundamentais para a formação dos elementos celulares que constituem a madeira.

\section{AGRADECIMENTOS}

Os autores agradecem ao Centro de Microscopia da Universidade Federal de Rio Grande e a Faculdade de Odontologia da Universidade Federal de Pelotas pela disponibilidade em realizar as análises de Difração de Raios-X, Microscopia Eletrônica de Varredura e Espectroscopia no Infravermelho. Também aos órgãos de fomento CAPES, CNPq e FAPERGS. 


\section{BIBLIOGRAFIA}

[1] SUN, Q. F., LU, Y., XIA, Y. Z., et al., "Flame retardancy of wood treatedby $\mathrm{TiO}_{2} / \mathrm{ZnO}$ coating", Surface Engineering, v. 28, n. 8, pp. 555-559, 2012.

[2] COPAT, G. P., Madeira tratada com ignífugos: análise da resistência mecânica após exposição ao fogo, Trabalho de conclusão de curso, Escola de Engenharia, Universidade Federal do Rio Grande do Sul, Porto Alegre, RS, Brasil, 2014.

[3] LEPAGE, E.S., Manual de preservação de madeiras. São Paulo, IPT/SICCT, 1986. 708p.

[4] PINTO, E. M., CALIL JÚNIOR, C., "Estudo teórico e experimental sobre a degradação térmica e os gradientes térmicos da madeira de Eucalyptus de uso estrutural exposta ao fogo", Revista Minerva, v. 3, n. 2, pp. 131-140, 2006.

[5] JIANG, J., LI, J., GAO, Q., "Effect of flame retardant treatment on dimensional stability and thermal degradation of wood", Construction and building materials, v. 75, pp. 74-81, 2015.

[6] OSTMAN, B., TSANTARIDIS, L., "Innovative eco-efficient high fire performance wood products for demanding applications”, Wood Technology, Stockholm, Sweden, 2006.

[7] LOWDEN, L. A., HULL, T. R., "Flammability behaviour of wood and a review of the methods for its reduction", Fire science reviews, v. 2, n. 1, 2013.

[8] BASSON, G. R., \& CONRADIE, W. E., U.S. Patent No. 6,319,431. Washington, DC: U.S. Patent and Trademark Office, 2001.

[9] UNER, I. H., DEVECI, I., BAYSAL, E., et al., "Thermal analysis of Oriental beech wood treated with some borates as fire retardants", Maderas, Ciencia y tecnología, v. 18, n. 2, pp. 293-304, 2016.

[10] SCHARTEL B., "Phosphorus-based flame retardancy mechanisms - old hat or a starting point for future development", Materials, v. 3, n. 10, pp. 4710-4745, 2010.

[11] WANG, X., LIU, J., CHAI, Y., "Thermal, mechanical, and moisture absorption properties of wood- TiO2 composites prepared by a sol-gel process", BioResources, v.7, n. 1, pp. 0893-0901, 2012.

[12] HABIBZADE, S., TAGHIYARI, H. R., OMIDVAR, A., et al., "Effects of impregnation with styrene and nano-zinc oxide on fire-retarding, physical, and mechanical properties of poplar wood", Cerne, v. 22, n. 4, pp. 465-474, 2016.

[13] COSTA, A. C. F. M., VILAR, M. A., LIRA, H. L., et al., "Síntese e caracterização de nanopartículas de $\mathrm{TiO}_{2}$ ", Cerâmica, v. 52, n. 324, 2006.

[14] PELAEZ, M., FALARAS, P., LIKODIMOS, V., et al., "Synthesis, structural characterization and evaluation of sol-gel-based $\mathrm{NF}^{-\mathrm{TiO}_{2}}$ films with visible light-photoactivation for the removal of microcystinLR", Applied Catalysis B: Environmental, v. 99, pp. 378-387, 2010.

[15] LEE, N. -H., OH, H. -J., JUNG, S. -C., et al., "Photocatalytic properties of nanotubular-shaped $\mathrm{TiO}_{2}$ powders with anatase phase obtained from titanate nanotube powder through various thermal treatments", International Journal of Photoenergy, v. 2011, p. 7, 2011.

[16] MURUGAN, A. V., SAMUEL, V., RAVI, V., "Synthesis of nanocrystalline anatase TiO2 by microwave hydrothermal method", Materials Letters, v. 60, 2006.

[17] BILECKA, I.; NIEDERBERGER, M., "Microwave chemistry for inorganic nanomaterials synthesis". Nanoscale, v.2, n.8, 2010.

[18] MOREIRA, M. L., Titanatos de alcalinos terrosos: a ordem associada à desordem, Tese de DoutoradoUniversidade Federal de São Carlos, São Carlos, SP, Brasil, 2010.

[19] SILVA, L. F., Síntese e caracterização do composto SrTiO3 e SrTil-xFexO3 através do método hidrotermal assistido por microondas. Tese de doutorado, Interunidades em Ciência e Engenharia de Materiais, Instituto de Física, São Carlos, SP, Brasil, 2013.

[20] KONG, L., TU, K., GUAN, H., et al., "Growth of high-density ZnO nanorods on wood with enhanced photostability, flame retardancy and water repellency”, Applied Surface Science, v. 407, pp. 479- 484, 2017.

[21] RASSAM, G., ABDI, Y., ABDI, A., "Deposition of $\mathrm{TiO}_{2}$ nano-particles on wood surfaces for UV and moisture protection”, Journal of Experimental Nanoscience, v. 7, n. 4, pp. 468-476, 2012.

[22] SUN, Q., LU, Y., ZHANG, H., et al., "Hydrothermal fabrication of rutile $\mathrm{TiO}_{2}$ submicrospheres on wood surface: an efficient method to prepare UV-protective wood", Materials Chemistry and Physics, v. 133, n. 1, pp. 253-258, 2012. 
[23] WANG, B., FENG, M., \& ZHAN, H., "Improvement of wood properties by impregnation with $\mathrm{TiO}_{2}$ via ultrasonic-assisted sol-gel process", RSC Advances, v. 4, n. 99, pp. 56355-56360, 2014.

[24] KUO, M.-L., MCCLELLAND, J. F., LUO, S., et al., "Applications of infrared photo-acoustic spectroscopy for wood samples”, Wood \& Fiber Science, v. 20, n. 1, p. 132-145, 1988.

[25] HESSE, M., MEIER, H. “Metodos Espectroscopicos En Quimica Organica”. Madrid: Sintesis Editorial, 2000.

[26] JIN, C., YAO, Q., LI, J., et al., "Fabrication, superhydrophobicity, and microwave absorbing properties of the magnetic $\gamma-\mathrm{Fe}_{2} \mathrm{O}_{3}$ /bamboo composites", Materials \& Design, v. 85, pp. 205-210, 2015.

[27] KIM, H.S, KIM, S., KIM, H.J., et al., "Thermal properties of bio-flour-filled polyolefin composites with different compatibilizing agent type and contente", Thermochimica Acta, v. 451, pp. 181- 188, 2006.

[28] LU, X., HU, Y., "Layer-by-layer deposition of $\mathrm{TiO}_{2}$ nanoparticles in the wood surface and its superhydrophobic performance", BioResources, v. 11, n. 2, pp. 4605-4620, 2016. 